\title{
Towards a Medical Oriented Social Network Service: Analysis of Instant Messaging Communication among Emergency Physicians
}

\author{
Kensuke Morris, ${ }^{*}, \#$ Osamu Sugryama, ${ }^{* *}$ Goshiro Yamamoto, ${ }^{* * *}$ Manabu Shimoto, ${ }^{\dagger}$ Genta Kato,${ }^{\dagger \dagger}$ \\ Shigeru Ohtsuru, ${ }^{\dagger}$ Masayuki Nambu, ${ }^{* *}$ Tomohiro Kuroda ${ }^{* * *}$
}

\begin{abstract}
The use of social network service (SNS) applications for health communication has revolutionized communication between physicians in recent years. We performed an unrestricted retrospective study focusing on emergency physicians (EPs) in Kyoto University Hospital (KUHP) since timely communication is important during emergencies. EPs used LINE, a popular SNS application in Japan. EPs $(n=22)$ sent 1752 messages from April 2017 to March 2018. Most messages sent contained text data (82.1\%), the remaining contained media (17.9\%); media included images (72.6\%), LINE stamps (22.9\%), LINE albums (2.3\%) and files $(1.6 \%)$. Content analysis by two coders produced 13 categories $(n=1438)$; these were 'miscellaneous', 'patient', 'team', 'treatment', 'event', 'situation', 'reference', 'announcement', 'schedule', 'resource', 'policy', 'transport' and 'unknown'. The top five message categories were related to miscellaneous chat $(22.5 \%)$, patient $(19.1 \%)$, team $(14.3 \%)$, treatment $(11.8 \%)$ and event $(6.6 \%)$. The largest number of messages among EPs were sent on Monday and Friday. The numbers of messages sent among EPs during day-shift and night-shift were similar. The categories identified influenced our proposal of medical oriented SNS platform features: structured tagging system for messages related to relevant categories (F1); inquiry broadcast system for specific inquiries using structured tagging (F2); image tagging system for images shared within groups (F3) and summarized notifications (F4). Features that need consideration are (1) an opt-in location sharing system between physicians and (2) physicians' access to patient records from the SNS application. In this study, messages discussed by EPs were categorized and the resulting categories influenced our proposal of a physician-centered SNS platform customized to EPs' roles. Since physicians prefer using SNS applications compared to traditional mobile phones, their information needs should be considered. Designing a medical oriented SNS platform that is physician-centered should first include an understanding of topics discussed by physicians. Based on the categories classified, the proposal of physician-centered features for designing a medical oriented SNS platform is also discussed in this paper.
\end{abstract}

Keywords: health communication, SNS application, emergency department, mHealth, SNS features.

Adv Biomed Eng. 9: pp. 35-42, 2020.

This study was presented at the Symposium on Biomedical Engineering 2019, Tokushima, September, 2019.

Received on July 23, 2019; revised on October 31, 2019 and December 23, 2019; accepted on December 25, 2019.

* Department of Social Informatics, Graduate School of Informatics, Kyoto University, Kyoto, Japan.

** Preemptive Medicine \& Lifestyle-Related Disease Research Center, Kyoto University Hospital, Kyoto, Japan.

*** Division of Medical Information Technology and Administration Planning, Kyoto University Hospital, Kyoto, Japan.

${ }^{\dagger}$ Department of Primary Care \& Emergency Medicine, Kyoto University Hospital, Kyoto, Japan.

${ }^{\dagger \dagger}$ Solutions Center for Health Insurance Claims, Kyoto University Hospital, Kyoto, Japan.

\# 54 Shogoin Kawahara-cho, Sakyo-ku, Kyoto 606-8507, Japan.

E-mail: kmorris@kuhp.kyoto-u.ac.jp

\section{Introduction}

Healthcare communication has improved with the advent of online social platforms [1]. Using online social platforms, physicians can communicate effectively with patients and other physicians [2]. Pervasive smartphone use has further transformed healthcare communication between physicians via Social Network Service (SNS) applications [3, 4]. In the emergency setting, physicians can transfer large amounts of data within short periods of time [5]. This is valuable because emergency physicians need timely and reliable information.

Despite the recent use of SNS applications for healthcare communication, analysis of communication between physicians using SNS applications has been reported [5-7]. Galacti et al. [5] and Jain et al. [6] reported 
the use of SNS applications by emergency and consulting physicians for consultation with patients. They retrospectively categorized physicians' communication data based on message type into images, text, video, and audio. Using a prospective approach, Johnston et al. [7] compared communication types and clinical domains by frequency of interactions between surgical teams using SNS. They also measured the interaction between various ranks of physicians and its frequency.

Such studies have provided valuable insights by analysis of healthcare communication between physicians using SNS applications. While Johnston et al. [7] categorized text data by clinical domain, data were not analyzed retrospectively, and the scope of the study did not include additional topics discussed by physicians. To the best of our knowledge, no study has categorized text data based on topics discussed by emergency physicians (EPs) using SNS applications.

This study analyzed text message communication topics discussed by EPs who used an SNS application during day- and night-shifts, and qualitatively categorized similar message topics that arose during these communications. The proposal of physician-centered feature specification towards designing an SNS platform is also discussed in this paper.

\section{Method}

\subsection{Study Design}

We performed a retrospective study focusing on EPs in Kyoto University Hospital (KUHP), for whom timely communication is important during emergencies. The personal accounts of EPs were added on a voluntary basis to a LINE group chat. LINE is an SNS application originally built to facilitate communication [8]. LINE became widely adopted in Japan with a variety of free and paid services; instant messaging and audio/video call services remain free. Previous studies used WhatsApp ${ }^{1}$ but we chose LINE because it is widely used in Japan and many EPs were not familiar with the former. EPs not physically present used LINE to communicate with EPs working in the Emergency Department (ED) during their shifts. This study was performed with approval from EPs. Due to the nature of this study, approval from an ethical committee was not required. EPs continued to use traditional mobile phones to discuss work-related matters on a one-to-one basis, i.e., an EP may make an inquiry on the group chat but discuss verbally with another EP a confidential matter relating to a patient.

\footnotetext{
${ }^{1}$ An SNS application which is popular in many countries. Similar to LINE, instant messaging and audio/ video calls are free. Also, chat groups can be created.
}

\subsection{Data Collection}

We extracted LINE data from April 2017 to March 2018. The LINE group is managed by EPs based on a professional guideline, which prohibits EPs from sending messages that contain patients' identifiable data, and the topics discussed were unrestricted in this professional SNS communication environment.

Prior to analysis, both the patient identifiable information and the messenger identifiable information, including those of the EPs, were anonymized by the data manager at ED and subsequently provided to third-party coders. Data contained no images of patients.

\subsection{Analysis}

Coders used content analysis to code the data (1752 lines) [9]. Each sentence was coded based on the topic represented. Two coders participated; both have a background in medical informatics. Coders coded messages into category names after mutual agreement. Context of similar codes was considered during categorization.

Categories were evaluated using Python version 3.5.2 (packages included pandas 0.24 .2 ; re 2.2.1; matplotlib 3.0.3). We evaluated the day(s) most messages were sent, the frequencies of communication per dayand night-shift, numbers of messages during day-shift, night-shift, weekdays and weekends. Day-shift is from $9 \mathrm{am}$ to $5 \mathrm{pm}$ with a transition period from 8 am to $9 \mathrm{am}$. Night-shift is from $5 \mathrm{pm}$ to 9 am with $\mathrm{T}$ from $4 \mathrm{pm}$ to $5 \mathrm{pm}$. Weekdays are Monday through Friday, and weekends are Saturday and Sunday.

\section{Results}

Twenty-two EPs sent 1752 messages between April 2017 and March 2018, with the majority containing text data $(82.1 \%)$ and the remaining containing media (17.9\%), as shown in Table 1. Media included images (72.6\%), LINE stamps (22.9\%), and LINE albums (2.3\%).

\subsection{Message categories}

The text data messages were classified into 13 categories as shown in Table 2. 'Miscellaneous' included any message unrelated to the professional role of emergency physicians. 'Patient' included messages in which a patient was mentioned directly or indirectly. 'Team' included messages about the emergency team, colleagues (inside and outside hospital) and issues, e.g. team shortages. 'Treatment' contained messages about treatment and medical procedures. 'Event' included messages about events such as meetings and conferences. 'Situation' included messages concerning a situation that occurred or is occurring. 'Reference' included messages with reference to printed or online resources such as medical guidelines and journals, that contained useful informa- 
tion to support discussions between physicians and/or treatment of patients.

'Announcement' included news, notices, updates about situations or resources. 'Schedule' included messages concerning EPs schedule and their availability during unexpected situations. 'Resource' contained messages regarding equipment or supplies used by EPs. 'Policy' included messages concerning rules, regulations,

Table 1 Characteristics of messages (1752).

\begin{tabular}{lll}
\hline \hline Variable & & Count [\%] \\
\hline Message Type & Text & $1438[82.1]$ \\
& Media & $314[17.9]$ \\
Media & & \\
& Image & $228[72.6]$ \\
& LINE stamp & $72[22.9]$ \\
& LINE albums & $7[2.3]$ \\
& Files & $5[1.6]$ \\
& Video & $1[0.3]$ \\
& LINE note & $1[0.3]$ \\
& & \\
Total messages & Day-shift & $832[47.5]$ \\
& Night-shift & $920[52.5]$ \\
& & \\
& Weekday & $1338[76.4]$ \\
& Weekend & $414[23.6]$ \\
\hline \hline
\end{tabular}

Table 2 Categories of text data messages (total 1438) sent among emergency physicians.

\begin{tabular}{lll}
\hline \hline & Message Category & Count [\%] \\
\hline 1. & Miscellaneous & $323[22.5]$ \\
2. & Patient & $274[19.1]$ \\
3. & Team & $205[14.3]$ \\
4. & Treatment & $170[11.8]$ \\
5. & Event & $95[6.6]$ \\
6. & Situation & $72[5.0]$ \\
7. & Reference & $63[4.4]$ \\
8. & Announcement & $60[4.2]$ \\
9. & Schedule & $50[3.5]$ \\
10. & Resource & $37[2.6]$ \\
11. & Policy & $27[1.9]$ \\
12. & Transport & $21[1.5]$ \\
13. & Unknown & $41[2.9]$ \\
\hline \hline
\end{tabular}

procedures or policies. 'Transport' included messages about any form of transport used for transporting patients or healthcare professionals. 'Unknown' included other messages not agreed upon by coders during categorization.

\subsection{Messages by time of communication}

A total of 1338 messages were sent on weekdays (76.4\%) and 414 during weekends (23.6\%). The highest number of messages sent was during the last weekday, Friday (23\%), and the second highest was during Mondays. Messages sent on Tuesdays and Wednesdays were about $50 \%$ fewer compared to Fridays; the messages sent on these days were also less than the numbers of messages sent during other weekends (Fig. 1).

The transition period $(\mathrm{T})$ was the hour preceding a main shift (M) when incoming physicians discussed matters with outgoing physicians. As shown in Fig. 2, the numbers of messages sent between one hour before and one hour after T (15 pm to $18 \mathrm{pm}, 7 \mathrm{am}$ to $9 \mathrm{am}$ ) were similar. Additionally, 119 messages were sent during $\mathrm{T}$ preceding night-shift, which was $53 \%$ more compared to $\mathrm{T}$ preceding day-shift. During night-shifts, the number of messages sent declined steadily after 19:00. No messages were sent between 2:00 and 4:00.

\subsection{Summary of results}

'Miscellaneous' messages were the most frequent $(18.4 \%)$. Although this category included messages unrelated to EPs' roles, it was included because the social environment shared by people in professional settings will involve communication unrelated to their profession. An SNS platform should not limit the conversations

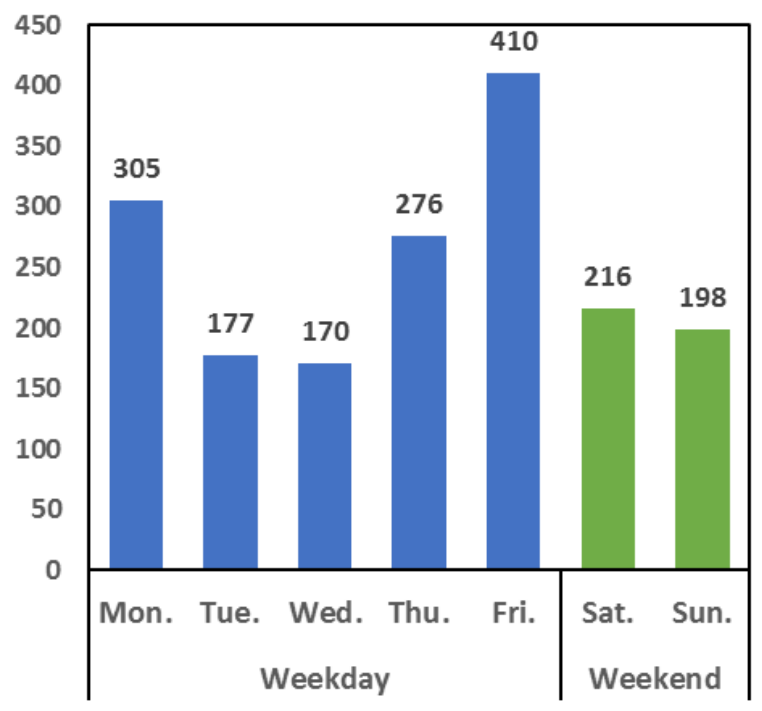

Fig. 1 Total number of messages sent during days of the week. 


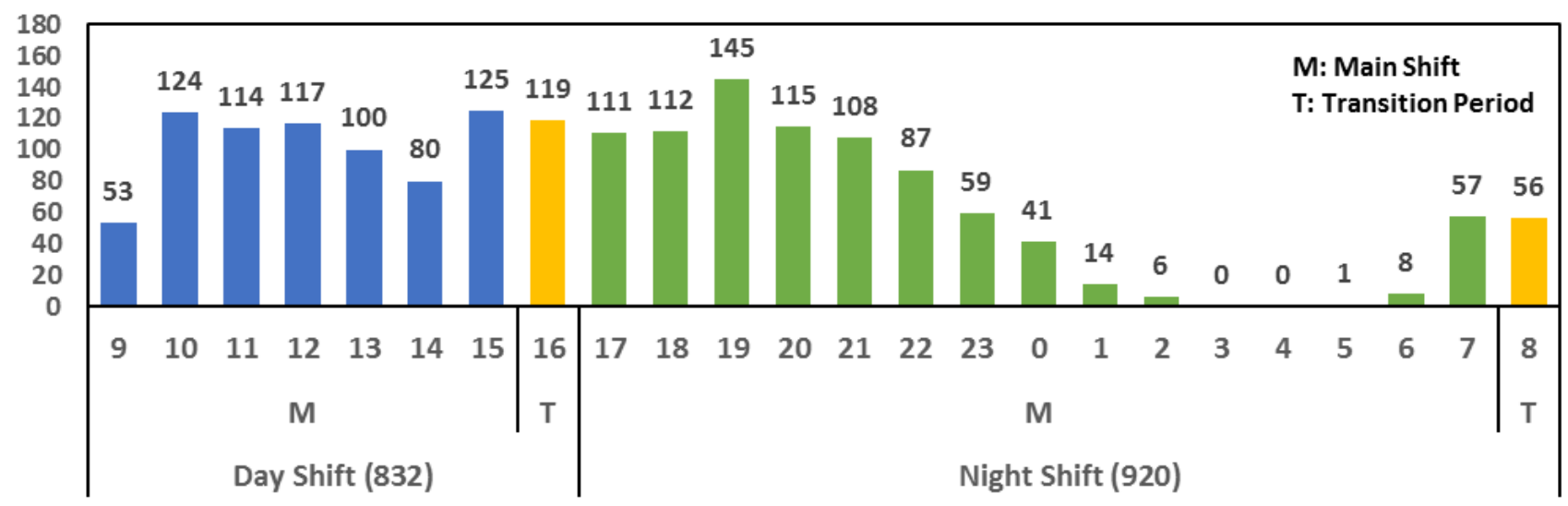

Fig. 2 Total number of messages (1752) per hour.

between physicians since they are by virtue of their job description bound by professional guidelines. The high frequency of 'Miscellaneous' messages suggests the importance of communication between physicians for purposes beyond their professional roles [10]. The focus of this study was to retrospectively categorize text messages, and the importance or implications of miscellaneous chat among physicians was outside the scope of this study.

'Media' messages comprised the second highest frequency (17.9\%). In consideration of an SNS platform for hundreds of physicians, it is important to prevent the existence of redundant images to improve the management, search and retrieval of large amounts of images. Additionally, some images are considered sensitive. Analysis of image contents was outside the scope of this study. As mentioned in section 2.2, in this retrospective study actual images were removed to safeguard the identities of patients. Future work will target the contents of images in a prospective study with approval from an ethics committee.

Some messages contained topics that overlapped, such as discussions about a patient (Category 2) including medical guidelines (Category 7) and treatment option (Category 4). Also, discussions about 'teams' sometimes referred to 'schedule'. Each category was deemed necessary in the context of medical emergencies, which is unpredictable.

To utilize an SNS system for communication between physicians, features within the SNS application should support day-to-day activities and discussions between physicians. In the case of EPs, these features should ensure that effective and efficient communication is achieved. The resulting categories suggest that an SNS application system is useful for EPs to facilitate both professional and informal conversation.

\section{Discussion}

The emergency domain often involves unpredictable scenarios. This study allowed us to obtain better understanding of how physicians in EDs use SNS applications to communicate. To design a user-centered system, it is important to first know the users and their preferences [11]. To the best of our knowledge, design specifications for a physician-centered SNS system are yet to be explored based on features derived from analysis of communication data among EPs. To date, there are no reported feature specifications that should be included in an SNS platform for physicians. Through this study, we are developing an understanding of EPs' communication using SNS applications by retrospectively categorizing their messages [12]. The present results were shown to and discussed with the EPs at KUHP.

\subsection{Medical oriented design}

This study is part of the design stage that precedes system development. The findings that contribute to our proposal of medical oriented SNS features are based on categories derived from physician-to-physician communication. For EPs, use of SNS applications has contributed to improved communication with colleagues $[5,7]$. Increased use of SNS among physicians suggests the necessity of physician-centered SNS platforms which contain features that supplement the physicians' roles. An SNS application designed for physicians should not increase their workload. By considering topics categorized from physicians' communications, designers can obtain an initial idea of which features may support inquiries and responses during physician-to-physician communication.

\subsection{Feature proposal}

Communication between EPs involves the sharing of information about various topics that are mainly related to 
EPs' roles. SNS features that consider EPs' roles can improve healthcare communication using SNS applications.

Considering the categories shown in section 3.1, discussions with EPs, and previous studies [5, 7, 12], we propose the following features: (F1) a structured tagging system for messages related to particular categories, (F2) an inquiry broadcast system for specific inquiries using F1, (F3) an image tagging system for images shared within groups, and (F4) summarized notifications. Familiarization with the data and the context surrounding the analyzed messages also influenced the coders' selection of these features.

\subsubsection{Structured tagging}

Based on the results listed in Table 2 and Fig. 1, F1 integrated in an SNS platform would help EPs navigate through large amounts of messages in a short time. This is not limited to healthcare communication using SNS applications but also includes social media websites [13]. Table 1 shows the categories that may form the basis of the structured tagging system. Using custom tags, physicians can create specialized tags that are based on formal and informal medical jargons used among medical teams. The tags would be easier to remember and adoptable for incoming physicians. According to Fig. 1, larger numbers of messages are sent among physicians on some days, and a structured tagging system can assist with retrieving messages sent previously.

Figure 2 suggests the usefulness of F1, since EPs on night-shift can conveniently retrieve messages sent during day-shift. The largest number of messages sent during the days was 58 . On that day, $72 \%$ of the messages were related to the 'patient' topic. F1 used with a detailed sub-category tagging system can assist physicians by filtering messages that may be about a 'treatment' option for a 'patient'. On a weekly basis, KUHP receives an average of 97 ambulance visits and treats an average of 229.4 patients. Although the number of participants in the present study was under 25, F1 can make the use of SNS applications more convenient for groups with larger numbers of physicians [14].

\subsubsection{Inquiry broadcast system}

F2 would provide physicians treating patients with access to feedback about treatment options. It could assist physicians by broadcasting inquiries tagged using F1 to reach the relevant doctors $[15,16]$. According to Figs. 1 and 2, physicians utilized LINE during weekends more than Tuesday and Wednesday. Additionally, LINE was utilized during night-shifts, suggesting that F2 can be used by physicians on weekends, night-shifts and national holidays to retrieve specific inquiries about topics not limited to digital resources and guidelines.

Current SNS group chats allow physicians to send specific inquiries which are seen by all group members, which is useful for information transparency within groups. However, there are cases where specific inquiries should be sent and discussed with specific physicians. Physicians should not have to use valuable time to check messages that are not related to them. F2 can improve efficient access to similar questions for medical students, interns, residents, fellow physicians who use smartphones [17]. This feature can be useful for tagging messages of related topics presented in section 3.1, especially 'patient', 'treatment', 'team', 'reference' and 'resource'. F2 can assist physicians in sending inquiries to more experienced physicians who may not be in the hospital at the time of inquiry $[18,19]$.

\subsubsection{Image tagging}

The findings in Table 1 show that images comprised $72.6 \%$ of media messages sent between EPs, suggesting the need for a system that allows efficient retrieval of images sent between physicians previously. F3 is needed to associate images with topics for future reference. $\mathrm{Al}-$ though F1 uses text to associate topics to messages, F3, in addition, will automatically describe the contents of images, which can provide a better association of relevant images to specific inquiries [20]. Using F3 combined with F1 and F2, EPs can save time when searching for information that is not related to their role of treating patients, and may contribute to reduction of non-clinical tasks [21, 22].

\subsubsection{Summarized notifications}

Summarized notifications are needed to prioritize messages since physicians need to be able to access important information in most relevant messages. LINE is preferred by physicians because of its simple user interface. However, the notification feature is not convenient for large group chats. As shown in Table 2, miscellaneous messages make up the largest category of messages $(18.4 \%)$. Although this category is useful, an immediate response may not be required. Thus, from the notification, emergency physicians can determine whether it is necessary to open certain messages during a busy shift. Although miscellaneous conversations may be useful for the team, this feature can limit miscellaneous messages that can be seen as interruptive to physicians' roles [23, 24]. F1 can contribute to summarized notifications from medical oriented SNS applications.

\subsubsection{Additional features}

Additional features for consideration are (1) an opt-in location sharing system and (2) access to patient records 
via the medical oriented SNS application. These considerations are mainly influenced by the 'patient', 'team', and 'situation' categories. Since the additional features may contribute to privacy concerns, additional guidelines and safeguards should be included for physicians who share their location, such as the option of revoking access to their location. Prior to a physician receiving requested information, they need to access the holder(s) of the relevant information [21]. During emergencies (day-to-day and after disasters), physicians needed at a certain point in time can be located by their colleagues. Since rules and regulations regarding the handling of patient data vary among countries, additional guidelines are needed to govern access to patients' clinical information via medical oriented SNS applications [25].

Kawai et al. [26] analyzed physicians' use of SNS for communication after natural disasters; their results led to the design and implementation of a feature integrated into an SNS application to support disaster medical assistance teams. During emergencies, such as a disaster aftermath, communication between physicians includes the use of medical information under certain guidelines. Using third-party communication platforms such as SNS applications, consideration must be given to how medical information is used [27] and shared among medical professionals. Additionally, during emergencies, physicians prefer communication tools that can be used beyond limitations of the internal hospital environment. Categories shown in section 3.1 and proposed features can address their preferences.

\subsection{SNS in Hospitals}

Integrating healthcare information that is user-centered is an ongoing challenge. Yu et al. [28] described characteristics of the healthcare domain towards improving interaction in EDs. Among the characteristics mentioned were non-routine, mobile, highly collaborative and information-rich. This study considers the mobile nature of EPs' roles where use of cellular phones, although convenient, is limited to two participants at a time. Additionally, although a third-party SNS application such as LINE or WhatsApp can provide group communication between physicians, the features available are not physician-centered. Physicians cannot communicate freely using third-party applications partly due to privacy concerns and current medical guidelines [29]. A physician-centered SNS platform that can support medical information sharing without using third-party platforms is more appropriate for the healthcare setting [27, 30].

An integrated SNS platform in hospital can revolutionize how EPs access to needed information using integrated SNS applications on mobile devices. This study focuses on improving physicians' communication, a sub- set of broader interactions that occur in the healthcare domain.

\subsection{Limitations}

This study focused on EPs in the medical emergency department of one hospital. However, to ensure flexibility of communication between EPs, a medical oriented SNS platform linking EPs in many hospitals is preferred. Furthermore, topics discussed by nurses and other health care professionals need to be investigated towards the design of an integrated SNS platform that considers various healthcare professionals.

\section{Conclusion}

Physicians' usage of SNS applications for health-related communication has increased recently and has revolutionized communication between physicians. In this retrospective study, we categorized 13 topics, based on messages exchanged between emergency physicians, and discussed their usefulness in the design a physician-centered SNS platform customized to the role of emergency physicians. The categories coded by two coders were 'miscellaneous', 'patient, 'team', 'treatment', 'event', 'situation', 'reference', 'announcement', 'schedule', 'resource', 'policy', 'transport' and 'unknown'. Mondays and Fridays were the days where most messages were sent between physicians.

The coded categories and number of messages sent in relation to shifts and days influenced our proposal of the following features: (F1) a structured tagging system for messages related to relevant categories, (F2) an inquiry broadcast system for specific inquiries using F1, (F3) an image tagging system for images shared within groups, and (F4) summarized notifications. Additional features for consideration include an opt-in physician location sharing system and (2) access to patient records via the medical oriented SNS application. The use of mobile SNS applications has improved healthcare communication between physicians. Improving the convenience of physicians' SNS discussions and access to information is possible using medical oriented SNS applications with SNS features designed in part by physicians, for physicians.

\section{Acknowledgement}

We express our special thanks to the emergency physicians at Kyoto University Hospital for their participation and cooperation throughout this study. Without their support, this study would not have been possible.

\section{References}

1. Moorhead SA, Hazlett DE, Harrison L, Carroll JK, Irwin A, Hoving C: A New dimension of health care: systematic review of the 
uses, benefits, and limitations of social media for health communication. J Med Internet Res. 15(4), e85, 2013.

2. Giustini DM, Ali SM, Fraser M, Boulos MNK: Effective uses of social media in public health and medicine: a systematic review of systematic reviews. Online J Public Health Inf. 10(2), 2018.

3. Mosa ASM, Yoo I, Sheets L: A systematic review of healthcare applications for smartphones. BMC Med Inf Decis Mak. 12(1), pp. 67, 2012.

4. Baig MM, GholamHosseini H, Connolly MJ: Mobile healthcare applications: system design review, critical issues and challenges. Australas Phys Eng Sci Med. 38(1), pp. 23-38, 2015.

5. Gulacti U, Lok U, Hatipoglu S, Polat H: An analysis of WhatsApp usage for communication between consulting and emergency physicians. J Med Syst. 40(6), 2016.

6. Jain S, Gupta M, Khuteta R: Social media messenger: useful tool for decision making in obstetric emergency duty hours. Int J Adv Res. 5(4), pp. 1582-1587, 2017.

7. Johnston MJ, King D, Arora S, Behar N, Athanasiou T, Sevdalis N, Darzi A: Smartphones let surgeons know WhatsApp: An analysis of communication in emergency surgical teams. Am J Surg. 209(1), pp. 45-51, 2015.

8. Line Corporation: Mexico earthquake - LINE to offer free international calls to landlines and mobile phones in Mexico 2014. <http://official-blog.line.me/en/archives/1006892897.html> [accessed on July 22, 2019]

9. Vaismoradi M, Turunen H, Bondas T: Content analysis and thematic analysis: Implications for conducting a qualitative descriptive study. Nurs Health Sci. 15(3), pp. 398-405, 2013.

10. Cameron AF, Webster J: Unintended consequences of emerging communication technologies: Instant messaging in the workplace. Comput Human Behav. 21(1), pp. 85-103, 2005.

11. Vaishnavi V, Kuechler W, Petter S: Design Science Research in Information Systems. <http://www.desrist.org/ design-research-in-information-systems/> [accessed on June 15, 2019]

12. Buchanan R: Wicked problems in design thinking. Des Issues, 8(2), p. 5-21, 1992.

13. White C, Plotnick L, Kushma J, Hiltz SR, Turoff M: An online social network for emergency management. Int J Emerg Manag. 6(3/4), pp. 369-382, 2009.

14. Ganasegeran K, Renganathan P, Rashid A, Al-Dubai SAR: The m-Health revolution: Exploring perceived benefits of WhatsApp use in clinical practice, Int J Med Inf. 97, pp. 145-151, 2017.

15. Lee S, Tang C, Park SY, Chen Y: Loosely formed patient care teams: Communication challenges and technology design. Proc of ACM Conference on Computer Supported Cooperative Work, ACM, New York, pp. 867-876, 2012.

16. Zhang AX, Cranshaw J: Making sense of group chat through collaborative tagging and summarization. Proc. of ACM Human-Computer Interact, Vol. 2, ACM, New York, pp. 1-27, 2018.

17. Payne KFB, Wharrad H, Watts K: Smartphone and medical related App use among medical students and junior doctors in the United Kingdom (UK): a regional survey. BMC Med Inf Decis Mak. 12(1), p. 121, 2012.

18. Fagin R, Halpern JY, Moses Y, Vardi MY: Common knowledge revisited. Ann Pure Appl Log. 96(1-3), pp. 283-298, 1996.

19. Halpern JY, Moses Y: Knowledge and common knowledge in a distributed environment. J ACM. 37(3), pp. 549-587, 1990.
20. Müller H, Michoux N, Bandon D, Geissbuhler A: A review of content-based image retrieval systems in medical applications Clinical benefits and future directions. Int J Med Inf. 73(1), pp. 1-23, 2004.

21. Ayatollahi H, Bath PA, Goodacre S: Information needs of clinicians and non-clinicians in the Emergency Department: a qualitative study. Heal Inf Libr J. 30(3), pp. 191-200, 2013.

22. Rogoski RR: IT in the ED. The nature of emergency department medicine means specific and comprehensive IT needs for clinicians. Health Manag Technol. 23(2), pp. 14-6, 2002.

23. Coiera EW, Jayasuriya RA, Hardy J, Bannan A, Thorpe MEC: Communication loads on clinical staff in the emergency department. Med J Aust. 176(9), pp. 415-418, 2002.

24. Berg LM, Källberg AS, Göransson KE, Östergren J, Florin J, Ehrenberg A: Interruptions in emergency department work: an observational and interview study. BMJ Qual Saf. 22(8), pp. 656663, 2013.

25. Whittaker R: Issues in mHealth: findings from key informant interviews. J Med Internet Res. 14(5), p. e129, 2012.

26. Kawai T, Kambara H, Matsumura K, Noma H, Sugiyama O, Shimoto M, Ohtsuru S, Kuroda T: Design and implementation of a social networking service-based application for supporting disaster medical assistance teams. Proc of International Conference on Innovation in Medicine and Healthcare. Vol. 71, Springer, Cham, pp. 165-172, 2018

27. Watson L, Pathiraja F, Depala A, O’Brien B, Beyzade S: Ensuring safe communication in health care: a response to Johnston et al on their paper 'Smartphones let surgeons know WhatsApp: an analysis of communication in emergency surgical teams'. Am J Surg. 211(1), pp. 302-303, 2016.

28. Yu E, Kealey R, Chignell M, Ng J, Lo J: Smarter healthcare: An emergency physician view of the problem. In: Smart Internet. Springer, Berlin, pp. 9-26, 2010

29. Nikolic A, Wickramasinghe N, Claydon-Platt D, Balakrishnan V, Smart P: The use of communication apps by medical staff in the australian health care system: survey study on prevalence and use. JMIR Med Inf. 6(1), p. e9, 2018.

30. McKechnie IEF: Updating NHS technologies: A WhatsApp-like system would improve communication. BMJ, Vol. 361. <https:// doi.org/10.1136/bmj.k1317> [accessed on September 20, 2019]

\section{Kensuke MORrIS}

Kensuke MORRIS received his B.Sc. in Information Technology from St. George's University in 2014 and obtained M.S. in Informatics from Kyoto University in 2017. He is currently pursuing his $\mathrm{PhD}$ in Informatics at the Graduate School of Informatics, Kyoto University and is also a student in the Kyoto University Collaborative Graduate Design Program.

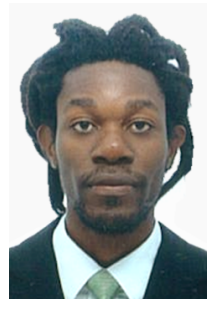




\section{Osamu SugiYama}

Osamu SugiYama received his PhD from School of Science for Open and Environmental Systems, Keio University in 2009. He is currently a Senior Lecturer in the Preemptive Medicine \& Lifestyle Related Disease Research Center, Kyoto University Hospital. His research fields are Medical Infor-

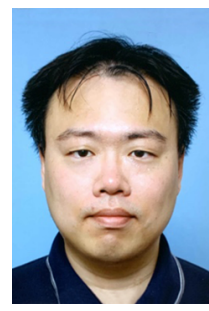
matics, Communication Robot, Robot Audition. His research interests are in machine learning utilizing medical information and health promotion with personal health records. Dr. SugIYAMA is currently managing and maintaining Data Warehouse System for supporting the research work in Kyoto University Hospital. He is affiliated with the Robotic Society of Japan, the Japanese Society for Artificial Intelligence and the Architectural Institute of Japan.

\section{Goshiro Yамамото}

Goshiro Yамамото received his BE, ME, and $\mathrm{PhD}$ in engineering from Osaka University. He is currently a Senior Lecturer in Kyoto University Hospital after being an Assistant Professor in Okayama University and Nara Institute of Science and Technology. His major research interest is $\mathrm{Hu}-$ man-Computer Interaction.

\section{Genta KaTO}

Genta Kato MD. PhD., completed the doctoral course from the Faculty of Letters, Kyoto University in 2012 and obtained his medical license in 1998. He is currently an Associate Professor at Kyoto University and Vice Director of the Solutions Center for Health Insurance Claims, Kyoto

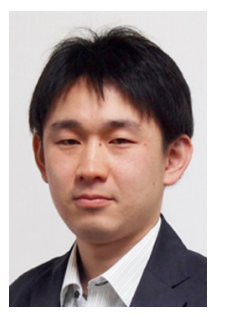
University Hospital. Dr. Kato also specializes in Emergency Medicine. His research focuses on health policies and discovering a system for handling large-scale health insurance claims database (NDB).

\section{Manabu Sнгмото}

Manabu Sнiмото MD. PhD., completed the doctoral course in Medicine at Kyoto University in 2017 and is currently working at the Department of Primary Care and Emergency Medicine at Kyoto University Hospital since 2011. He is also an Assistant Professor in the Graduate School of Medi-

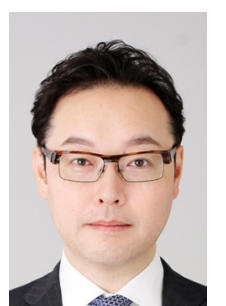
cine and he specializes in Emergency and Disaster Medicine. Dr. Sнгмото is member of Japanese Association of Acute Medicine and Japanese Association for Disaster Medicine.

\section{Shigeru Oнtsuru}

Shigeru Ohtsuru MD. PhD., completed the doctoral course in Medicine at Kyoto University in 2014. He graduated from Kobe University School of Medicine in 1998. He is currently an Associate Professor in the Graduate School of Medicine, Kyoto University. He is also the Vice Chairman

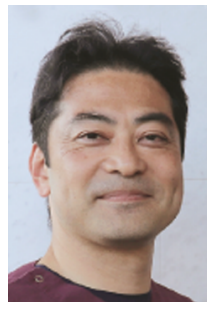
and Medical Office Manager of the Department of Primary Care and Emergency Medicine, Kyoto University Hospital. Additionally, Dr. OHtsuru is also an Associate Professor at the Section of Health Emergency and Regional Disaster Risk Management, Disaster Prevention Research Institute, Kyoto University.

\section{Masayuki NAMBU}

Masayuki NAmBU received Ph.D. degree from Nara Institute of Science and Technology in 1999. He is currently Program-Specific Professor, in Preemptive Medicine \& Lifestyle Related Disease Research Center. His research interests include biomedical measurement and signal processing,

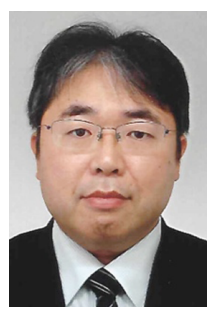
image processing, telecare and home care technology. He is currently a member of Japanese Society for Medical and Biological Engineering. He is also a member of IEEE EMBS.

\section{Tomohiro KURODA}

Tomohiro KURODA received B.S. in information science from Kyoto University, Japan in 1994, M.S. and Ph.D. in information science from Nara Institute of Science and Technology, Japan in 1996 and 1998. He was with Graduate School of Information Science at Nara Institute of Science and

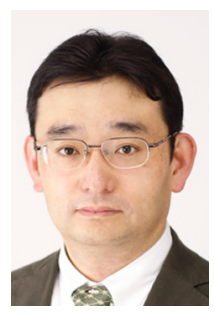
Technology, Japan from 1998 to 2001, where he held the rank of assistant professor, with Department of Medical Informatics of Kyoto University Hospital, Japan from 2001 to 2006 and from 2009 to 2013, where he held the rank of lecturer and associate professor respectively, and with Department of Information Processing Science of University of Oulu, Finland in 2001 and 2006, where he held the rank of visiting professor. He was with Graduate School of Engineering Science of Osaka University, Japan from 2007 to 2009, where he held the rank of associated professor. Since August 2013, he has been the director of Division of Medical Information Technology and Administrative Planning of Kyoto University Hospital, Japan, where he also serves as the professor of medical informatics in the graduate school of medicine and the graduate school of informatics. His current research interests include Human Interface, Virtual/Augmented Reality, Wearable/Ubiquitous Computing, and Medical/Assistive informatics. He is a member of IEEE, ISVR, VRSJ, HISJ, JSMBE, JAMI, JSMVR, ISCIE, JASL, and others. 